\title{
Protée
}

\section{La photographie aérienne, l'échelle, le point de vue}

\section{Anne Beyaert-Geslin}

Volume 37, numéro 3, hiver 2009

Regards croisés sur les images scientifiques

URI : https://id.erudit.org/iderudit/038805ar

DOI : https://doi.org/10.7202/038805ar

Aller au sommaire du numéro

Éditeur(s)

Département des arts et lettres - Université du Québec à Chicoutimi

\section{ISSN}

0300-3523 (imprimé)

1708-2307 (numérique)

Découvrir la revue

Citer cet article

Beyaert-Geslin, A. (2009). La photographie aérienne, l'échelle, le point de vue. Protée, 37(3), 57-64. https://doi.org/10.7202/038805ar

\section{Résumé de l'article}

L'article observe en quoi les notions de point de vue et d'échelle déterminent la signification de la photographie aérienne. Il montre comment un pays prend forme, sa présence iconique (la constitution d'une forme) s'alliant nécessairement à une présence référentielle (la localisation de cette forme sur terre). d'utilisation que vous pouvez consulter en ligne.

https://apropos.erudit.org/fr/usagers/politique-dutilisation/ 


\title{
LA PHOTOGRAPHIE AÉRIENNE, L'ÉCHELLE, LE POINT DE VUE
}

\author{
ANNE BEYAERT-GESLIN
}

\section{INTRODUCTION}

L'étude de l'image scientifique ouvre de vastes perspectives à la sémiotique visuelle. D'un côté, elle permet de reformuler des questions qui, si elles n'étaient mises en relation avec les genres et les statuts, risqueraient de rester confinées dans la généralité et l'approximation. Elle révèle plus précisément que la signification d'une image est nécessairement déterminée par une pratique et accessible à une communauté précise: à chaque pratique scientifique, correspond un usage signifiant des images. D'un autre côté, le fait qu'elle interroge les limites du visible l'amène à problématiser la question de l'iconicité. Elle dévoile ainsi l'instabilité des phénomènes, les aléas de leur inscription et les exigences de leur transformation en une forme stabilisée et identifiable. Sur ce point, la photographie aérienne s'avère particulièrement intéressante et, par la projection de grandeurs extrêmes, engage à reconsidérer les questions de point de vue et d'échelle. Selon la distance du point de vue, l'inscription des phénomènes est en effet différente et stabilise une forme plus ou moins identifiable. Une nouvelle présence iconique se construit, dont les paramètres suivent une transformation graduelle mais subissent aussi des sauts qualitatifs qui mobilisent différents niveaux de sens.

Pour commencer, la distance opère une transformation épistémologique, la modification de l'apparence de l'objet induisant le renouvellement de l'instance d'observation. Il faut aussi tenir compte des exigences particulières des usages sociaux qui imposent que ces inscriptions de phénomènes soient non seulement stabilisées, mais aussi suffisamment identifiables pour être partagées et "pratiquées " dans la communauté qui en fera usage.

Notre étude se consacrera aux questions de point de vue et d'échelle. Elle s'attachera à divers corpus correspondant à des "versions différentes d'un seul et même monde neutre et sous-jacent» (Goodman, 2006: 40) qui, établissant des règles de corrélation différentes entre les valeurs au sens terrestre, telles qu'elles sont consacrées par les routines perceptives, et les valeurs au sens céleste, construisent des objets de sens distincts.

\section{LES VARIATIONS DU POINT DE VUE}

En toute première approximation, les photographies prises à partir du ciel se laissent décrire comme des images construites pour autant qu'elles sont des 
orthophotographies. Projeter l'objet courbe qu'est la surface terrestre sur les deux dimensions d'une image oblige en effet à homogénéiser l'échelle au moyen d'une correction focale obtenue par projection de la hauteur sur la largeur du plan. Or, dès l'abord, cette orthocorrection introduit des variantes dans l'image en fonction de son destinataire, ses paramètres différant selon qu'elle relève de la communication scientifique ou de la vulgarisation. Lorsque le destinataire est un scientifique, l'image pratique l'orthocorrection mathématique et soumet le monde naturel à sa visée géométrique; lorsqu'elle s'adresse au grand public, elle reste en revanche au plus près de la perception du piéton qui pourra ainsi confronter ces données nouvelles à son expérience quotidienne. Ces pratiques et ces destinataires distincts correspondent à deux acceptions de l'exactitude cartographique telle que la définit Bertin (1998). Elles instaurent des rapports différents à la vérité qui n'excluent pas un certain paradoxe, puisque l'image la plus correcte au sens de la géométrie s'avère la plus déroutante lorsqu'elle est rapportée aux usages de la perception et apparaît donc comme la moins réaliste au sens de Goodman (1968). La vérité mathématique s'oppose à la vérité perceptive.

À l'orthocorrection, premier critère de construction du plan de l'expression, s'ajoute celui de la distance qui décline trois familles de photographies selon la grande, la moyenne ou la faible altitude. Les photographies les plus distanciées sont prises à partir des satellites; celles qui empruntent les distances et échelles moyennes sont des vues d'avion. Cependant, loin de se résumer à une question d'instrument conditionné par la distance, une telle distinction implique un accès différent au visible. Lorsque l'altitude est très faible, l'hypothèse d'une perspective à vol d'oiseau peut en effet laisser place à une perspective en hauteur qui situe le point de vue, non pas au-dessus de la surface terrestre, mais au niveau de sa plus grande élévation. Si leur hypothèse topologique semble offrir une plus grande diversité d'images (perspective à vol d'oiseau ou en hauteur), les vues d'avion peuvent également être considérées comme plus précises parce qu'elles accèdent au visible comme à l'invisible et peuvent associer «couleurs vraies" et "couleurs fausses». En effet, confrontées à la perception directe et au monde visible, elles utilisent les "couleurs vraies" conformes à notre expérience du monde naturel; cependant, un dispositif infrarouge peut permettre de repousser les limites du monde visible par la projection de «fausses couleurs» qui viendront affiner la distinction entre les zones humides et sèches, par exemple. À plus grande distance, la seule possibilité de percevoir, d'enregistrer et de stabiliser les inscriptions des phénomènes en une forme identifiable ${ }^{1}$ est donnée par la lumière infrarouge. L'image est alors prise à partir d'un satellite et s'obtient par une conversion des données sensibles en données chromatiques. Ces valeurs, qui peuvent tout aussi bien restituer des données thermiques qu'hydrométriques ou restituer un objet invisible à l'œil nu (la longueur d'onde de ces couleurs se situe au-delà et en deçà de 0,4 et $0,8 \mu^{2}$ ), se transforment alors en un plan d'expression constitué par superposition de différentes couches de «couleurs fausses».

Ainsi esquissée, cette typologie construite sur la notion de distance fournit plusieurs critères de construction du plan de l'expression. En effet, si la distance détermine les limites du monde visible, ellesmêmes présidant au choix de couleurs dites «vraies" ou "fausses», elle introduit un second axe sémantique, le rapport au vivant ou à l'animé. À faible distance, l'échelle humaine reste valide et peut accompagner certaines manifestations de la vie organique, suivre le déplacement d'un troupeau ou d'un vol d'oiseaux, par exemple. C'est d'ailleurs à faible distance que la mobilité du point de vue s'avère la plus pertinente, car elle permet de révéler ce principe d'animation. En revanche, les photographies prises à haute altitude n'ont accès à la vie animale ou humaine qu'au moyen des "fausses» couleurs de l'infrarouge ("fausses vraies» ou «vraies fausses» selon qu'elles ressemblent à celles de la perception directe), l'accès à l'animé supposant alors une intrusion dans le caché. Cette stratification du visible permet d'inférer une modification de l'instanciation. En effet, à faible distance, l'énonciation préserve une instance subjective et la figure d'un auteur. L'énonciateur est le plus souvent un narrateur qui «raconte» la terre peuplée d'actants 
livrés à des épreuves et argumente sur des effets de sens conformes aux valeurs mythiques ${ }^{3}$. À plus grande distance, cette énonciation devient nécessairement impersonnelle et technique, c'est celle d'un oilmachine contrôlé par l'avion ou le satellite.

\section{OÙ COMMENCE L'IMAGE SCIENTIFIQUE}

Cette relation entre la distance et l'instanciation doit être conservée en mémoire comme un acquis de l'analyse de la photographie aérienne susceptible d'être soumis à un nouvel examen pour chaque genre d'image et chaque pratique, fût-elle scientifique ou non. Ainsi, à propos d'un corpus de photos de reportage (Beyaert-Geslin, 2009), avons-nous mis en évidence un contraste entre les deux "extrémités" de l'événement. La plus petite et la plus grande distance, où la première sollicite une écriture corporelle - le frottement du corps somatique, caractéristique des photographies granuleuses du débarquement en Normandie de Robert Capa, par exemple -, mais virtualise l'activité de sujet, tandis que les photographies prises à la plus grande distance, dites d'actualité, virtualisent, au contraire, le corps somatique et sollicitent un énonciateur, en tant que subjectivité, devant reconsidérer toutes les dimensions de l'énonciation et assurer la sélection des figures textuelles et des genres de photographies (portrait, nature morte...). Pour la photographie aérienne, cette instanciation particulière laisse apparaître un point de basculement où l'image reconsidère les termes de la semiosis. Elle bascule d'un régime de témoignage où le plan d'expression se confond avec les possibilités de la perception directe, vers un régime d'imagerie où l'apparence d'une expérience sensible est associée à une expérience scientifique (Fontanille, 2007) pour construire un nouveau système semi-symbolique. Ce point d'émergence coïncide avec l'utilisation d'instruments qui, telles des prothèses, démultiplient les capacités perceptives, et convertit l'exposition du visible des données en une exploration de l'invisible (ibid.).

\section{OÙ L'CEIL PERD SES REPÈRES}

Ces prémices permettent de comprendre comment, devant renouveler l'instanciation et les modalités d'accès au visible, la photographie aérienne établit une nouvelle semiosis et construit un nouvel objet de sens. Une telle description ne saurait éluder la question de l'échelle car, lorsque la photographie aérienne recourt aux instruments, elle perd aussi tous les repères familiers qui assurent la maîtrise de la forme et son ancrage dans le monde sensible. Plus exactement, la hauteur perd la référence à la stature humaine avec laquelle nous organisons les valeurs dans le monde visible et dans les images. Ce repère, posé dès Protagoras, n'a cessé d'être confirmé jusqu'à Simmel qui définit la figure humaine comme une norme permettant de mesurer le monde:

Quel que soit l'environnement dans lequel elle est placée, [la figure humaine] est ressentie comme la norme qui détermine les quantités et proportions de ce qui l'entoure. À l'intérieur d'une image donnée, elle-même n'est donc ni grande ni petite, puisqu'elle est bien plutôt ce à quoi se mesure la grandeur ou la petitesse de tous les autres éléments. (2003: 50)

Un peu d'attention permettrait de préciser ce point en mobilisant un argument corollaire. En effet, si la figure humaine permet d'aborder le monde, elle est aussi une échelle à partir de laquelle nous pouvons construire des mondes, comme l'explique Levi-Strauss (1969: 33) à propos du modèle réduit et de tableaux de Clouet. La stature humaine permet d'aborder l'existant, mais aussi d'inventer, de faire exister des mondes. Si l'on en croit Simmel, la perte de l'étalon de mesure serait d'autant plus dommageable que la hauteur fait aussi disparaître les objets de petites dimensions et nous confronte aux plus volumineux, les montagnes, les fleuves et les mers, dont la représentation reste la plus problématique. Pour «révéler leur sens et leur importance» (Simmel, 2003: 50), les objets doivent nécessairement être représentés selon une certaine échelle; cependant, si l'on peut sans dommage modifier l'échelle des objets "organiques» (la figure humaine ou l'arbre), les objets «inorganiques», telles les montagnes, exigent que leurs dimensions soient préservées parce que leur sens tient essentiellement à leur démesure, explique-t-il. Si aucun paysage alpin n'est vraiment convaincant, c'est parce que «l'attrait visuel des Alpes est dû essentiellement au caractère extraordinaire de leur masse, et leur 
valeur formelle ne révèle son efficacité esthétique qu'à cette échelle» (ibid.: 43). En somme, l'insertion d'un promeneur sur une image de dune permet d'identifier le paysage (c'est une photo de dune et non la macrophotographie d'un bac à sable) et, par inférence, de localiser la hauteur du point de vue (la plus haute élévation terrestre et non celle de mon corps lorsque je vise le sol). En perdant ce repère, la distance diversifie le sens et le livre à la polysémie. Le paysage est alors un morceau de croûte terrestre qui, tel le tableau de Max Ernst, L'Europe après la pluie (1933), autorise tous les investissements imaginaires. Lorsque l'échelle humaine est perdue, une difficulté supplémentaire apparaît qui tient à l'ontologie et à la méréologie du paysage. À la différence du corps humain, toujours susceptible d'être reconstruit à partir de ses parties, le paysage n'obéit pas à un schéma préétabli. Il se renouvelle sans cesse, n'est que différence, et cette propriété, qui fait l'agrément de la promenade, suffit à hypothéquer sa maîtrise conceptuelle et révèle l'importance particulière de la figure humaine.

Ces descriptions trop allusives devront être problématisées et exemplifiées dans la suite de cette étude. À ce stade, il importe surtout de noter que, lorsque l'échelle est toute petite, l'identification devient impossible et, comme l'indique Bertin, «le document est perdu pour l'information" (1998: 287). Faisant pendant avec l'échelle humaine, repère pour la plus faible distance, il importe de mobiliser le repère du planisphère qui convient à la plus grande distance, sachant que "plus l'espace est grand, plus il semble que la forme extérieure de la carte suffise à son identification" (ibid.). À l'échelle du pays, peu de formes restent reconnaissables et leur identification reste aléatoire et essentiellement culturelle ${ }^{4}$.

\section{VÉRITÉ ICONIQUE ET RÉFÉRENTIELLE}

Nous avons tâché de mettre l'image aérienne en mouvement pour révéler les variations du point de vue et activer les deux repères extrêmes, la figure humaine et celle du planisphère. Avec ces éléments, nous commençons à comprendre comment des instruments construisent les images et délimitent précisément leurs fonctions. Il reste à décrire les règles de construction de l'image (couleurs, formes, textures) et à relier celles- ci à une énonciation qui permet de les "pratiquer» relativement à un usage signifiant. Trivialement, le changement de distance de la photographie aérienne pose un problème lié au basculement du visible dans l'invisible. Lorsqu'on s'éloigne du sol, non seulement l'unité de l'objet de sens se défait (l'iconicité est compromise), mais celui-ci devient aussi incommensurable et son expérience ne peut plus être partagée (la symbolicité est mise en cause).

Deux solutions s'offrent alors qui tiennent compte des particularités de la pratique. La première, adoptée pour le site de l'annuaire des pages jaunes/blanches, consiste à intégrer au dispositif de référencement externe aux images (légendes, échelles de couleurs...), caractéristique des pratiques de vulgarisation, un point de mire sur lequel l'observateur pourra «zoomer» pour s'approcher jusqu'au niveau de la maison ou, en élargissant le champ, retrouver la distance de la rue, du quartier, de la ville, etc. Les images ainsi compactées permettent alors d'instaurer une chaîne de références où les inscriptions de phénomènes, en préservant une constance déictique, restituent la continuité de la transformation de la forme. L'objet géographique est ainsi conservé dans son unité. Il conserve la mémoire de toutes les transformations effectuées et chacune des étapes peut être retrouvée et répétée.

Ainsi conçu, ce processus d'aller et retour pour s'approcher ou s'éloigner du point de mire n'est pas sans rappeler l'enchaînement caractéristique des images scientifiques, dont l'effort vise à reconstituer une unité d'objet, comme l'a indiqué Maria Giulia Dondero (2009) à propos des images d'astrophysique. En compactant ces images, le site de l'annuaire permet d'écraser les discontinuités de la prise de distance, substituant alors aux références à la stature humaine et au planisphère une autoréférence qui fait de chaque image la référence de la suivante et, alors que l'absence de repères laisse craindre une perte d'information dans les images prises à distance intermédiaire, conserve la même densité d'un bout à l'autre de la chaîne de références.

Un autre dispositif, utilisé pour les images fixes mais également pour le site de l'annuaire qui associe les photos aériennes à un plan d'accès, consiste à 
transformer la photographie en une pseudo carte par l'ajout d'une configuration argumentative associant des références et un dispositif chromatique. Dans ce cas, la colorisation permet de constituer un plan d'expression en distribuant les valeurs selon un dispositif discret ou graduel. Selon l'usage social, la distribution des couleurs observera le principe contrastif et discret du plan du site de l'annuaire qui s'efforce de guider le voyageur de la façon la plus efficace, c'est-à-dire par une économie de moyens et de couleurs et une claire distinction des localités. Elle établit une règle de conversion chromatique fondée sur la plus grande différence chromatique, c'est-à-dire l'écart des couleurs primaires essentiellement. Dans d'autres cas, la distribution des couleurs suit au contraire un principe associatif et scalaire qui permet, en introduisant de faibles différences tonales, d'ordonner des informations tout en affinant le dessin du paysage. C'est le cas de la carte de la mer du Japon de l'Organisation hydrographique internationale, dont Tufte (2007: 76-77) explicite les avantages. Par la juxtaposition, cette carte autorise une fine distinction des zones immergées et émergées. Par une comparaison avec une autre carte qui décline toutes les couleurs de l'arc-enciel, Tufte montre que la gradualité tonale permet d'introduire une plus grande quantité d'informations tout en préservant la cohérence isotopique qui autorise les investissements cognitifs. D'où sa constatation: "d'infimes différences permettent plus de différences» (ibid.: 77 ; notre traduction).

Avec l'utilisation des couleurs, s'impose ainsi la nécessité de transformer les inscriptions de phénomènes en une forme stabilisée (iconicité), mais aussi identifiable, commensurable, exploitable et partageable (symbolicité), conforme au principe d'efficacité de Bertin:

[...] si, pour obtenir une réponse correcte et complète à une question donnée, et toutes choses égales, une construction requiert un temps d'observation plus court qu'une autre construction, on dira qu'elle est plus efficace pour cette question. $(1998: 146)^{5}$

\section{CONSTRUIRE L'IMAGE}

Mais la colorisation n'est pas la seule condition de l'exploitation de l'image. Pour que son usage soit signifiant, celle-ci doit encore être intégrée à une configuration argumentative finalisée par la pratique sociale, qui la stabilise et assure son exploitation, comme le montrera l'étude d'un atlas de vulgarisation rassemblant des photographies aériennes de la France.

La particularité de cet ouvrage est en effet de permettre l'identification de photographies qui, dégagées de ce support éditorial, échapperaient totalement à la signification. Comme nous l'avons indiqué précédemment, la particularité des stratégies éditoriales des documents de vulgarisation tient à la densité du dispositif textuel ajouté à l'image, extérieurement à l'image (légendes, barres de références chromatiques). Dans le cas de l'atlas de vulgarisation, un dispositif référentiel approprié permet à chaque page d'identifier précisément l'image aérienne en y associant le nom du pays ou de la région. Les références résolvent des problèmes d'identification qui se laissent au demeurant aborder par la question sémiotique de l'iconicité, mais qui, pour le lecteur, se traduisent simplement par une difficulté à circonscrire une figure dans une continuité paysagère. Très grossièrement, on pourrait avancer que représenter un pays revient à donner forme à un territoire et à produire la croyance iconique qui lui donnera consistance. Comment l'ouvrage parvient-il à produire cette croyance et à transformer une empreinte à peu près informe, perdue pour la sémiotique, en une forme stable, susceptible d'être identifiée et partagée par les lecteurs les moins avertis (vulgarisation)? C'est la question qui nous intéresse désormais.

Deux chapitres permettent de préciser le problème et la solution apportée par les auteurs de l'atlas. La difficulté la plus considérable intervient sans doute avec les photographies du Limousin qui, si elles ne subissaient la pression cartographique en s'autorisant quelques indications de lieux, ne «signifieraient» rien parce qu'elles seraient dénuées de toute présence iconique et restitueraient une belle continuité d'un vert sombre. Or, tandis que se construit la présence référentielle à partir d'une carte de France assortie d'un dispositif de cadres renvoyant à un index des territoires, une présence iconique se construit tout de même au fil des pages qui donne peu à peu 
consistance à une Région Limousin. Tout d'abord, des frontières administratives sont circonscrites au moyen d'un contraste chromatique et tonal (vert sombre sur blanc) qui interrompt les continuités paysagères entre le Limousin et les régions périphériques. Ce contraste superficiel permet de séparer une figure sur laquelle se concentrera l'attention, distincte d'un fond pouvant être négligé (Groupe $\mu, 1992)$. Bien que banal, ce traitement de l'image serait superflu en d'autres cas. Placé au bord de la mer, le Limousin recevrait dès l'abord un statut de figure et c'est donc le caractère continental de la région qui impose ce traitement. La mer fonctionne en effet comme un fond dans la photographie aérienne, un statut que lui confèrent certaines propriétés propres ou qui tiennent plutôt au contraste qu'elle oppose à la terre. Avant tout, elle oppose à la diversité chromatique de la terre une continuité contrastante, fût-elle décrite en noir ou en bleu. Ensuite, le bleu étant perçu comme la couleur la plus éloignée dans la profondeur, il tend à "pousser» les couleurs terrestres «devant» lui. Enfin, les contrastes de texture eau/terre tendent à marquer les différences chromatiques si bien que, comme l'explique Ninio, même si les lignes n'existent pas dans la nature, «la ligne de côte qui sépare l'eau de la terre est vue, de bateau ou d'avion, comme si elle avait été expressément dessinée à l'encre noire» (1996: 65).

À ces "carences iconiques", imputables au caractère continental de la région, s'ajoutent des difficultés liées non plus à la délinéation d'un contour, mais à la structuration de la plage. Les discontinuités, qui font le charme du paysage limousin vu du sol et donnent prise à la signification, s'évanouissent lorsqu'on les observe du ciel et laissent place à une granularité, à cette répétition d'éléments qui caractérise la texture (Beyaert-Geslin, 2003). En l'occurrence, la photographie restitue très fidèlement le monde sensible et ne propose aucune conversion chromatique susceptible d'améliorer la différenciation. Elle traduit le délicat modelé de cette basse montagne par une continuité texturale qui devient signifiante avec la médiation du cadre argumentatif de la cartographie et, notamment, la projection de noms de lieux.

Ces difficultés révélées par les pages de l'atlas consacrées au Limousin, et habilement palliées par les stratégies éditoriales, suffisent à révéler les conditions de la présence iconique et montrent que l'inscription des phénomènes donne lieu à des formes plus ou moins identifiables selon la morphologie et l'ontologie du paysage rencontré. Un peu d'attention révèle que l'identification géographique tend à privilégier la structure culturelle du paysage pour se concentrer sur les aéroports ou les autoroutes, par exemple, qui prennent une importance considérable pour l'orientation et la structuration du lieu, surtout dans le cadre des pratiques de vulgarisation. L'identification géographique interroge aussi sa structure naturelle, les lignes des fleuves et de la mer, les aspérités et les couleurs du relief, en fonction des besoins de la pratique. Elle s'appuie plus précisément sur une gamme chromatique qui, même lorsqu'elle réplique les «vraies couleurs», celles de l'expérience sensible, renvoie à une distribution des valeurs inhabituelle, réservée au positionnement élevé du point de vue. En transformant les différences d'altitude et de culture en contrastes chromatiques - en vert pour les plaines et les zones basses, en brun pour les zones intermédiaires et en blanc pour la cime des montagnes -, la gamme chromatique de la photographie aérienne permet de structurer le paysage dans ses deux ou ses trois dimensions.

Si cette rapide description suffit à révéler les «carences iconiques» de la photographie aérienne du Limousin qui, parce qu'il est dévolu à la nature et à une altitude moyenne, se traduit par une texture et non par des figures lorsqu'on l'aperçoit du ciel, une comparaison avec une région littorale et industrielle montre à l'inverse comment une présence iconique s'impose d'elle-même en raison des propriétés du terrain. Dans son survol de la Côte-d'Opale, l'atlas montre par exemple comment la mer assume le contraste figure/fond et l'accentue même par un effet de contour. Il révèle de même l'incidence de l'appropriation économique des lieux qui, assurant la variété chromatique, vient informer le plan de l'expression. Deux paramètres s'allient dès lors dans la stabilisation de la forme, un objet de sens se détachant pour ainsi dire de lui-même en même temps qu'il s'offre comme un champ d'investigation au regard. L'iconisation s'impose comme un processus 
global, assurant la "prise» de la forme, et comme un processus local qui introduit des inégalités signifiantes à l'intérieur de cette forme. Par ce double processus, seulement déterminé par le caractère littoral, industriel et urbanisé de la région, une présence iconique se construit en même temps que la vérité référentielle, parce que la localisation du territoire sur le littoral apporte aussi son concours à la stabilisation iconique.

Ce constat permet de faire l'hypothèse d'une corrélation entre la présence iconique et la présence référentielle dans la photographie aérienne, par laquelle la forme se laisse identifier en même temps qu'elle est localisée sur un territoire. Traduite par les critères tensifs, il s'agit d'une corrélation converse imposant la progression parallèle des grandeurs et l'augmentation de l'intensité (présence iconique) avec l'étendue (présence référentielle). Pourtant, cette esquisse doit être nuancée en fonction de la hauteur du point de vue, qui introduit des paliers de reconnaissance et de référenciation liés aux deux attracteurs iconiques extrêmes que sont la figure humaine et celle du planisphère. Notre réflexion aboutit à l'hypothèse selon laquelle l'identification des formes croise deux échelles référentielles. Une première échelle de référence, discontinue, subit la pression des deux attracteurs iconiques et permet d'identifier l'objet en le rapportant à ces grandeurs. Une seconde échelle, continue et indépendante de la hauteur, associe la vérité iconique et la vérité référentielle pour donner consistance à l'objet géographique construit en fonction de sa localisation sur la surface terrestre.

\section{POUR CONCLURE SANS CONCLURE}

Avant de conclure ce parcours, une remarque méthodologique s'impose. Dans notre effort pour conserver le fil de la démonstration, nous avons en effet unifié toutes les pratiques de l'image aérienne dans une conception synthétique qui gomme toutes leurs exigences spécifiques. Il convient donc maintenant de prendre nos distances vis-àvis d'une pensée monologique et de faire un lien explicite entre les pratiques scientifiques et les usages signifiants. Dans une étude consacrée aux dessins d'un programme de restauration d'une ouvre d'art ${ }^{6}$, nous avions montré que chaque genre d'image modélise l'objet qu'il aborde et opère un recentrage sémantique des propriétés de l'objet en les subordonnant à la finalité de la pratique. Chaque image se concentre sur un sens pratique précis et, animée d'un souci d'efficacité, opère une sélection des données pertinentes et des données «parasites». Mais la modélisation va bien au-delà de la simple interaction entre un informateur et un observateur, entre un destinateur et un destinataire, et même au-delà du recentrage sur un sens pratique. L'image prend en charge le processus de fictionnalisation de l'objet pour l'établir en fait visuel et assurer une modalisation épistémique. Ces différents résultats révélés par le modus operandi d'une pratique spécifique, la restauration d'une sculpture contemporaine, pourraient être réexaminés et utilisés à propos des images aériennes. Ils montreraient que les différents utilisateurs de cette image observée jusqu'ici de façon générique, les géographes mais aussi les climatologues ou les océanographes, reconfigurent l'image selon les besoins spécifiques de leur pratique, de sorte qu'une même photo pourra signifier pour l'un et pas pour l'autre, et même qu'une donnée pertinente pour l'un sera considérée comme parasite pour l'autre. Ainsi les graduations fines des profondeurs marines, indispensables à l'océanographe, serontelles considérées comme parasites pour le lecteur de notre atlas de vulgarisation qui, ayant déjà bien du mal à localiser son Limousin et devant discriminer les formes de la terre, exigera que la mer soit un simple fond, une continuité bleue. 


\section{NOTES}

1. On obtient alors une résolution supérieure qui permet de distinguer des zones de $2,5 \mathrm{~m}$ au sol, les dispositifs militaires actuels pouvant quant à eux distinguer des zones de $15 / 20 \mathrm{~cm}$.

2. La notion de monde visible est discutée dans le premier chapitre de l'ouvrage de Jacques Fontanille, Sémiotique du visible, intitulé "Comment le sens vient à la lumière " (1995).

3. Les différents films qui s'efforcent d'alerter sur les dangers du réchauffement climatique (Yann Artus Bertrand) situent le point de vue à faible distance du sol, ce qui donne accès au monde animé (animaux, mouvements des rivières...) et préserve une figure de narrateur. Cette double instanciation permet de faire le récit de la terre, de construire un parcours narratif.

4. Pour Bertin, certaines formes de pays pourraient être identifiées par tout le monde parce qu'elles «ont atteint un degré d'universalisation qui les hausse au niveau du symbole" (1998: 287). On objecterait que cette identification est aussi largement culturelle, l'habitant d'un pays parvenant à l'identifier même si ses formes sont compliquées.

5. L'efficacité se mesurant à la rapidité, on pourrait avancer que l'intentionnalité est ici de fournir une image, c'est-à-dire «une forme significative perceptible dans l'instant minimum de vision, perceptible spontanément" (Bertin, 1998: 146).

6. L'article, «Le dessin dans un programme de restauration d'une œuvre d'art» (Beyaert-Geslin, à paraître), restitue une communication faite lors de journées d'étude organisées à Venise en décembre 2008 dans le cadre de l'ANR «Images et dispositifs de visualisation scientifiques " (2008-2010) qui réunit Limoges, Liège, Venise et Strasbourg.

\section{RÉFÉRENCES BIBLIOGRAPHIQUES}

BEAutier, F. [2004]: La France vue de l'espace, Bagneux, Sélection du Reader's Digest.

BERTIN, J. [(1967)1998]: Sémiologie graphique. Les diagrammes, les réseaux, les cartes, Paris, École des hautes études en sciences sociales. BEYAERT-GesLin, A. [2003] : "Texture, couleur, lumière et autres arrangements de la perception ", Protée, vol. 31, n³, 81-90;

[2008]: "Paysage et catégories topologiques", actes du colloque Le Paysage. En ligne: http://revues.unilim.fr/nas/document. php?id=2456 (page consultée le 22 octobre 2009);

- [2009]: L'Image préoccupée. Sémiotique de la photographie de reportage, Paris, Hermès-Lavoisier;

[à paraître]: «Le dessin dans un programme de restauration d'une œuvre d'art", Visible, $\mathrm{n}^{\circ} 7$.

DONDERO, M. G. (2009]: «L'image scientifique: de la visualisation à la mathématisation et retour». En ligne: http://revues.unilim.fr/nas/ document.php?id=2907 (page consultée le 22 octobre 2009). FONTANILlE, J. [1995]: Sémiotique du visible. Des mondes de lumière, Paris, PUF;

[2003]: «Lumières, matières et paysages ", Protée, vol. 31, n 3, $17-30$

[2007]: «Les systèmes d'imagerie scientifique. Questions sémiotiques", Rivista dell Associazione italiani di studi semiotici. En ligne: http://www.ec-aiss.it/archivio/tipologico/autore.php (page consultée le 22 octobre 2009).

GOODMAN, N. [(1968) 2005]: Langages de l'art, une approche de la théorie des symboles, trad. de J. Morizot, Paris, Hachette;

[(1978) 2006]: Manières de faire des mondes, trad. de M.-D. Popelard, Paris, Gallimard.

GROUPE [1992]: Traité du signe visuel, Paris, Seuil.

JACOBI, D. [1999]: La Communication scientifique. Discours, figures, modèles, Grenoble, Presses universitaires de Grenoble.

LATOur, B. [(1999) 2001]: L'Espoir de Pandore. Pour une version réaliste de l'activité scientifique, Paris, La Découverte.

LeVI-Strauss, C. [1969]: La Pensée sauvage, Paris, Plon.

Ninio, J. [1996]: L'Empreinte des sens. Perception, mémoire, langage, Paris, Odile Jacob.

SimmeL, G. [2003]: "La quantité esthétique», Le Cadre et autres essais, trad. de K. Winkelvoss, Paris, Gallimard.

TUFTE, E. R. [(1997) 2007]: Visual Explanations, Images and Quantities, Evidence and Narrative, Cheshire (CT), Graphics Press. 